\title{
Partial Generalized Probability Weighted Moments for Exponentiated Exponential Distribution
}

\author{
Neema Mohamed El Haroun \\ Institute of Statistical Research \\ Mathematical Statistics Department \\ Cairo University, Egypt \\ na3omaalharoun@yahoo.com
}

\begin{abstract}
The generalized probability weighted moments are widely used in hydrology for estimating parameters of flood distributions from complete sample. The method of partial generalized probability weighted moments was used to estimate the parameters of distributions from censored sample. This article offers new method called partial generalized probability weighted moments (PGPWMs) for the analysis of censored data. The method of PGPWMs is an extended class from partial generalized probability weighted moments. To illustrate the new method, estimation of the unknown parameters from exponentiated exponential distribution based on doubly censored sample is considered. PGPWMs estimators for right and left censored samples are obtained as special cases. Simulation study is conducted to investigate performance of estimates for exponentiated exponential distribution. Comparison between estimators is made through simulation via their biases and mean square errors. An illustration with real data is provided.
\end{abstract}

Keywords: Generalized Probability Weighted Moments, Partial Probability Weighted Moments, Partial generalized Probability Weighted Moments, Generalized Exponential Distribution, Censored Samples.

\section{Introduction}

Gupta and Kundu (1999), introduced the generalized exponential distribution which, also known as exponentiated exponential (EE) distribution. It is observed that the $\mathrm{EE}$ distribution can be considered for situation where a skewed distribution for a nonnegative random variable is needed. Also, it is observed that it can use quite effectively to analyze lifetime data in place of gamma, Weibull and log-normal distributions.

The probability density function of the two parameter EE distributions given by;

$$
f(x ; \alpha, \lambda)=\alpha \lambda\left(1-e^{-\lambda x}\right)^{\alpha-1} e^{-\lambda x}, \quad \alpha, \lambda, x>0
$$

The corresponding cumulative distribution function is given by:

$$
F(x ; \alpha, \lambda)=\left(1-e^{-\lambda x}\right)^{\alpha}, \quad \alpha, \lambda, x>0
$$

The probability weighted moments are useful for estimating the unknown parameters and quantiles of a distribution from complete sample. Wang (1990a) introduced extended class of probability weighted moments which called partial probability weighted moments (PPWMs) to estimate the parameters of a distribution from censored sample. Wang (1990a, b, 1996) originally introduced that concept for the purpose of estimating the upper quantiles of flood flows when one's interest is in the right tail of the distribution and there is some benefit to censoring some of the smaller observations in the left tail. 
The initial idea of PPWMs was to remove the smaller observations from the process of fitting the distribution because such observations are of little interest in flood frequency analysis and such observations can a nuisance to the fitting process. The method is computationally simple, robust and possesses the same merit as the original method of probability weighted moments.

Zafirakou-Koulouris et al (1998) introduced the evaluation of L- moments for a left censored observation from PPWMs. Deng and Pandey (2009) presented a new distribution for estimating quantile function of a non-negative random variable using a censored sample of data, which is based on the principle of partial maximum entropy in which PPWMs are used as constraints.

This article presents new method which is called partial generalized probability weighted moments for estimating the unknown parameters of distributions from censored samples. The PGPWMs will be used to estimate unknown parameters of EE distribution under doubly censored samples. Then, the PGPWMs estimators of the unknown parameters from left and right censored samples will be obtained as special cases. At the same time, the generalized probability weighted moments can be obtained as the special case from PGPWMs. To illustrate the properties of the new estimators, an extensive numerical study will be performed. Analysis of a real data set has been performed.

This article is organized as follows. In Section 2, the definition of PGPWMs and their sample estimators from doubly, right, and left censored samples is introduced. Section 3 discusses the PGPWMs method of estimation for the EE distribution from doubly, left and right censored samples. A simulation study is performed to investigate the properties of the new estimators from censored samples with two special cases in Section 4. Real data example is given in Section 5. Finally, the conclusions are included in Section 6. Tables and some Figures are included in the appendix.

\section{The PGPWMs and Their Sample Estimators}

The generalized probability weighted moments (GPWMs) were introduced by Rasmussen (2001) as a tool for estimating the parameters of probability distribution, for complete sample, expressible in inverse form. The GPWMs can be defined as

$$
M_{p, u, v}=E\left(X^{p}\{F(X)\}^{u}\{1-F(X)\}^{v}\right)=\int_{0}^{1}\{x(F)\}^{p} F^{u}(1-F)^{v} d F,
$$

where $X$ is a random variable and $F(x)$ is cumulative distribution function.

We introduce the method of PGPWMs as an extension class of GPWMs and PPWMs methods. The general form of PGPWMs with double bound censoring for the random variable $\mathrm{X}$ is defined as follows:

$$
W_{p, u, v}=E\left(X^{p}\{F(X)\}^{u}\{1-F(X)\}^{v}\right)=\int_{c}^{d}\{x(F)\}^{p} F^{u}(1-F)^{v} d F,
$$


where, $p=1, u$ and $v$ to be real values (i.e. exponents $u$ and $v$ of the PGPWMs can be ratio or integers). Also, $c=F\left(x_{01}\right)$ and $d=F\left(x_{02}\right), x_{01}$ and $x_{02}$ are lower and upper bound censoring respectively; Therefore, for $p=1$, and $v=0, W_{p, r, s}$ becomes;

$W_{1, u, 0}=E\left(X\{F(X)\}^{u}\right)=\int_{c}^{d}\{x(F)\} F^{u} d F$,

It is noted when $d=1$ and the lower bound $c=0$ then the PGPWMs with double bound censoring is reduced to GPWMs in complete samples as defined in (2).

The PGPWMs with lower bound (left) censoring, denoted by $L W_{1, u, 0}$, can be obtained as a special case from (4)by putting $d=1$. Therefore, the general form of PGPWMs with left censoring for the random variable $\mathrm{X}$ takes the following form

$L W_{1, u, 0}=E\left(X\{F(X)\}^{u}\right)=\int_{c}^{1}\{x(F)\} F^{u} d F$.

The PGPWMs with upper bound (right) censoring, denoted by $R W_{1, u, 0}$, can be defined as a special case from (4) by putting $c=0$. Therefore, the general form of PGPWMs with right censoring for the random variable $\mathrm{X}$ takes the following form

$R W_{1, u, 0}=E\left(X\{F(X)\}^{u}\right)=\int_{0}^{d}\{x(F)\} F^{u} d F$.

Let $x_{(1)}, x_{(2)}, \ldots, x_{(n)}$ be a random sample of size $n$ from the distribution function $F(X)$, and $x_{(1)}<x_{(2)}, \ldots<x_{(n)}$ be the corresponding ordered sample. According to Hosking (1986), the estimates of $W_{1, u, 0}, L W_{1, u, 0}$ and $R W_{1, u, 0}$ is defined as the following

$$
\begin{aligned}
& \hat{W}_{1, u, 0}=\sum_{i=1}^{n} x_{(i)}\left(\frac{i-0.35}{n}\right)^{u} . \\
& L \hat{W}_{1, u, 0}=\sum_{i=1}^{n} x_{(i)}\left(\frac{i-0.35}{n}\right)^{u} . \\
& R \hat{W}_{1, u, 0}=\sum_{i=1}^{n} x_{(i)}\left(\frac{i-0.35}{n}\right)^{u} .
\end{aligned}
$$

Note that, the PGPWM estimators with doubly, left or right censoring is obtained by equating the theoretical PGPWM with the corresponding sample PGPWM.

\section{PGPWMs Estimation of the EE}

In this section, the PGPWMs estimation method will be used to estimate the unknown parameters from EE distribution with doubly censored samples. Furthermore, the PGPWMs estimators with left and right censored samples are obtained as special cases. 


\section{(3.1) PGPWMs Estimation of the EE from Doubly Censored Sample}

The theoretical PGPWMs with double bound censored, $W_{1, u, 0}$ for the EE distribution can be found from (4) after setting $u=u_{1}$ and $u=u_{2}$ as the following;

$$
\begin{aligned}
& W_{1, u_{1}, 0}=\int_{c}^{d}\{x(F)\} F^{u_{1}} d F=\frac{1}{\lambda} \sum_{j=1}^{\infty}\left[\frac{d^{\frac{j}{\alpha}+u_{1}+1}-c^{\frac{j}{\alpha}+u_{1}+1}}{j\left(\frac{j}{\alpha}+u_{1}+1\right)}\right] \\
& W_{1, u_{2}, 0}=\int_{c}^{d}\{x(F)\} F^{u_{2}} d F=\frac{1}{\lambda} \sum_{j=1}^{\infty}\left[\frac{d^{\frac{j}{\alpha}+u_{2}+1}-c^{\frac{j}{\alpha}+u_{2}+1}}{j\left(\frac{j}{\alpha}+u_{2}+1\right)}\right]
\end{aligned}
$$

From Equations (10) and (11), the two parameters, $\alpha$ and $\lambda$, can be expressed as follows:

$$
\begin{aligned}
& \lambda=\frac{\sum_{j=1}^{\infty}\left[\frac{d^{\frac{j}{\alpha}+u_{1}+1}-c^{\frac{j}{\alpha}+u_{1}+1}}{j\left(\frac{j}{\alpha}+u_{1}+1\right)}\right]}{W_{1, u_{1}, 0}} . \\
& W_{1, u_{2}, 0} \sum_{j=1}^{\infty}\left[\frac{d^{\frac{j}{\alpha}+u_{1}+1}-c^{\frac{j}{\alpha}+u_{1}+1}}{j\left(\frac{j}{\alpha}+u_{1}+1\right)}\right]-W_{1, u_{1}, 0} \sum_{j=1}^{\infty}\left[\frac{d^{\frac{j}{\alpha}+u_{2}+1}-c^{\frac{j}{\alpha}+u_{2}+1}}{j\left(\frac{j}{\alpha}+u_{2}+1\right)}\right]=0 .
\end{aligned}
$$

The PGPWMs estimators of $\alpha$ and $\lambda$ from doubly censored samples, can be obtained by solving equations (12) and (13) in terms of $\alpha$ and $\lambda$; where $W_{1, u_{1}, 0}$ and $W_{1, u_{2}, 0}$ are replaced by their sample estimators, $\hat{W}_{1, u_{1}, 0}$ and $\hat{W}_{1, u_{2}, 0}$ given by Equation (7) by setting $u=u_{1}, u_{2}$, therefore

$$
\begin{aligned}
& \hat{\lambda}=\frac{\sum_{j=1}^{\infty}\left[\frac{d^{\frac{j}{\hat{\alpha}}+u_{1}+1}-c^{\frac{j}{\hat{\alpha}}+u_{1}+1}}{j\left(\frac{j}{\hat{\alpha}}+u_{1}+1\right)}\right]}{\hat{W}_{1, u_{1}, 0}} . \\
& \hat{W}_{1, u_{2}, 0} \sum_{j=1}^{\infty}\left[\frac{d^{\frac{j}{\hat{\alpha}}+u_{1}+1}-c^{\frac{j}{\hat{\alpha}}+u_{1}+1}}{j\left(\frac{j}{\hat{\alpha}}+u_{1}+1\right)}\right]-\hat{W}_{1, u_{1}, 0} \sum_{j=1}^{\infty}\left[\frac{d^{\frac{j}{\hat{\alpha}}+u_{2}+1}-c^{\frac{j}{\hat{\alpha}}+u_{2}+1}}{j\left(\frac{j}{\hat{\alpha}}+u_{2}+1\right)}\right]=0,
\end{aligned}
$$

Solving numerically Equation (15) based on some iteration technique to obtain $\hat{\alpha}$. Once the estimate of $\alpha$ is determined, an estimate of $\lambda$ is obtained by substitute $\hat{\alpha}$ in Equation (14). 
Some special cases can be obtained from the above the PGPWMs

1. For $u_{1}=0$ and $u_{2}=1$, the PPWMs can be obtained from PGPWMs with doubly censored as obtained by Al-Khodary et al (2008).

2. For $c=0$ and $d=1$, the GPWMs can be obtained from PGPWMs with doubly censored as obtained by El Haroun (2009).

3. For $u_{1}=0, u_{2}=1, c=0$ and $d=1$, the PWMs can be obtained from PGPWMs with doubly censored as obtained by Al-Khodary et al (2007).

\section{(3.2) PGPWMs Estimation of the EE from Left Censored Sample}

The theoretical PGPWMs with left bound censored, $L W_{1, u, 0}$ for the EE distribution can be found from (5) after setting $u=u_{1}$ and $u=u_{2}$ as the following;

$$
\begin{aligned}
& L W_{1, u_{1}, 0}=\int_{c}^{1}\{x(F)\} F^{u_{1}} d F=\frac{1}{\lambda} \sum_{j=1}^{\infty}\left[\frac{1-c^{\frac{j}{\alpha}+u_{1}+1}}{j\left(\frac{j}{\alpha}+u_{1}+1\right)}\right] \\
& L W_{1, u_{2}, 0}=\int_{c}^{1}\{x(F)\} F^{u_{2}} d F=\frac{1}{\lambda} \sum_{j=1}^{\infty}\left[\frac{1-c^{\frac{j}{\alpha}+u_{2}+1}}{j\left(\frac{j}{\alpha}+u_{2}+1\right)}\right]
\end{aligned}
$$

From Equations (16) and (17), the two parameters, $\alpha$ and $\lambda$, can be expressed as follows:

$$
\begin{aligned}
& \lambda=\frac{\sum_{j=1}^{\infty}\left[\frac{1-c^{\frac{j}{\alpha}+u_{1}+1}}{j\left(\frac{j}{\alpha}+u_{1}+1\right)}\right]}{L W_{1, u_{1}, 0}} . \\
& L W_{1, u_{2}, 0} \sum_{j=1}^{\infty}\left[\frac{1-c^{\frac{j}{\alpha}+u_{1}+1}}{j\left(\frac{j}{\alpha}+u_{1}+1\right)}\right]-L W_{1, u_{1}, 0} \sum_{j=1}^{\infty}\left[\frac{1-c^{\frac{j}{\alpha}+u_{2}+1}}{j\left(\frac{j}{\alpha}+u_{2}+1\right)}\right]=0 .
\end{aligned}
$$

To compute the PGPWMs estimators of $\alpha$ and $\lambda$, denoted by $\hat{\alpha}_{L}$ and $\hat{\lambda}_{L}$, from left censored samples, $L W_{1, u_{1}, 0}$ and $L W_{1, u_{2}, 0}$ are replaced by their sample estimators, $L \hat{W}_{1, u_{1}, 0}$ and $L \hat{W}_{1, u_{2}, 0}$ by setting $u=u_{1}, u_{2}$ in Equation (8), hence

$$
\hat{\lambda}_{L}=\frac{\sum_{j=1}^{\infty}\left[\frac{1-c^{\frac{j}{\hat{\alpha}_{L}}+u_{1}+1}}{j\left(\frac{j}{\hat{\alpha}_{L}}+u_{1}+1\right)}\right]}{L \hat{W}_{1, u_{1}, 0}},
$$




$$
L \hat{W}_{1, u_{2}, 0} \sum_{j=1}^{\infty}\left[\frac{1-c^{\frac{j}{\hat{\alpha}_{L}}+u_{1}+1}}{j\left(\frac{j}{\hat{\alpha}_{L}}+u_{1}+1\right)}\right]-L \hat{W}_{1, u_{1}, 0} \sum_{j=1}^{\infty}\left[\frac{1-c^{\frac{j}{\hat{\alpha}_{L}}+u_{2}+1}}{j\left(\frac{j}{\hat{\alpha}_{L}}+u_{2}+1\right)}\right]=0
$$

Solving numerically by iteration Equation (20) to obtain $\hat{\alpha}_{L}$. Once the estimate of $\alpha$ is determined, an estimate of $\lambda$ is obtained by substitute $\hat{\alpha}_{L}$ in Equation (21).

\section{(3.3) PGPWMs Estimation of the EE from Right Censored Sample}

The theoretical PGPWMs with right bound censored, $R W_{1, u, 0}$ for the EE distribution can be obtained from (6) after setting $u=u_{1}$ and $u=u_{2}$ as the following

$$
\begin{aligned}
& R W_{1, u_{1}, 0}=\int_{0}^{d}\{x(F)\} F^{u_{1}} d F=\frac{1}{\lambda} \sum_{j=1}^{\infty}\left[\frac{d^{\frac{j}{\alpha}+u_{1}+1}}{j\left(\frac{j}{\alpha}+u_{1}+1\right)}\right] \\
& R W_{1, u_{2}, 0}=\int_{0}^{d}\{x(F)\} F^{u_{2}} d F=\frac{1}{\lambda} \sum_{j=1}^{\infty}\left[\frac{d^{\frac{j}{\alpha}+u_{2}+1}}{j\left(\frac{j}{\alpha}+u_{2}+1\right)}\right]
\end{aligned}
$$

From Equations (22) and (23), the two parameters, $\alpha$ and $\lambda$, can be expressed as follows:

$$
\begin{aligned}
& \lambda=\frac{\sum_{j=1}^{\infty}\left[\frac{d^{\frac{j}{\alpha}+u_{1}+1}}{j\left(\frac{j}{\alpha}+u_{1}+1\right)}\right]}{R W_{1, u_{1}, 0}} . \\
& R W_{1, u_{2}, 0} \sum_{j=1}^{\infty}\left[\frac{d^{\frac{j}{\alpha}+u_{1}+1}}{j\left(\frac{j}{\alpha}+u_{1}+1\right)}\right]-R W_{1, u_{1}, 0} \sum_{j=1}^{\infty}\left[\frac{d^{\frac{j}{\alpha}+u_{2}+1}}{j\left(\frac{j}{\alpha}+u_{2}+1\right)}\right]=0 .
\end{aligned}
$$

The PGPWMs estimators of $\alpha$ and $\lambda$, denoted by $\hat{\alpha}_{R}$ and $\hat{\lambda}_{R}$, from right censored samples, can be obtained by solving Equations (24) and (25); where $R W_{1, u_{1}, 0}$ and $R W_{1, u_{2}, 0}$ are replaced by their sample estimators, $R \hat{W}_{1, u_{1}, 0}$ and $R \hat{W}_{1, u_{2}, 0}$ by setting $u=u_{1}, u_{2}$, in Equation (9) as follows

$$
\hat{\lambda}_{R}=\frac{\sum_{j=1}^{\infty}\left[\frac{d^{\frac{j}{\hat{\alpha}_{R}}+u_{1}+1}}{j\left(\frac{j}{\hat{\alpha}_{R}}+u_{1}+1\right)}\right]}{R \hat{W}_{1, u_{1}, 0}},
$$




$$
R \hat{W}_{1, u_{2}, 0} \sum_{j=1}^{\infty}\left[\frac{d^{\frac{j}{\hat{\alpha}_{R}}+u_{1}+1}}{j\left(\frac{j}{\hat{\alpha}_{R}}+u_{1}+1\right)}\right]-R \hat{W}_{1, u_{1}, 0} \sum_{j=1}^{\infty}\left[\frac{d^{\frac{j}{\hat{\alpha}_{R}}+u_{2}+1}}{j\left(\frac{j}{\hat{\alpha}_{R}}+u_{2}+1\right)}\right]=0 .
$$

A numerical procedure is required to estimate $\alpha$ based on iterative technique. Thus the value of $\hat{\lambda}_{R}$ can be obtained by substituting the value of $\hat{\alpha}_{R}$ in Equation (27).

\section{Numerical Experiments and Discussions}

An extensive numerical study is carried out to investigate the properties of the PGPWMs method of estimation for the EE distribution from censored samples. The investigated properties are biases and mean square error (MSE) of the PGPWMs estimators of the two parameters $\alpha$ and $\lambda$. The simulation procedure can be summarized as the following steps:

Step (1): 1000 random sample, $x_{1}, x_{2}, \ldots, x_{n}$, of sizes $n=15,20,25,30,35$ and 50 are generated from the EE distribution.

Step (2): $\quad$ The true parameter selected values for the shape parameter $\alpha$ are $0.5,0.6$, 0.7 with scale parameter $\lambda=1$. Choosing the exponent values of PGPWMs as $u_{1}=0,0.1,0.5$ and $u_{2}=1,0.4,1.5$. Levels of censoring are considered, namely $c=0.2$ and $d=0.8$.

Step (3): $\quad$ For each combination of values of sample size $\mathrm{n}, \alpha$ and $\lambda$, the parameters of distribution are estimated using three different estimation methods; PGPWMs with doubly, left and right censored samples.

Step (4): $\quad$ PGPWMs of the unknown parameter $\alpha$ for EE distribution with doubly censored samples are obtained by solving numerically the non-linear Equation (15). The estimate of the scale parameter $\lambda$ is obtained by substituting the value of $\hat{\alpha}$ in (14).

Step (5): $\quad$ Based on left censored samples, PGPWMs of the shape parameter $\alpha$ for EE distribution is obtained by solving iteratively the non-linear Equation (21). Hence the $\hat{\lambda}_{L}$ is obtained by substituting the value of $\hat{\alpha}_{L}$ in (20)

Step (6): $\quad$ Based on right censored samples, PGPWMs of the shape parameter $\alpha$ for EE distribution is obtained by solving numerically the non-linear Equation (27). To compute $\hat{\lambda}_{R}$, substitute $\hat{\alpha}_{R}$ in (26)

Step (7): $\quad$ PPWMs estimators with doubly, left and right censored samples for $\alpha$ for EE distribution is obtained by solving numerically the non-linear Equations (15),(21) and (27) after setting $u_{1}=0$ and $u_{2}=1$. Then, the estimates of $\lambda$ is obtained by substituting the estimate of $\alpha$ in Equations (14), (20) and (26).

Step (8): $\quad$ The MSE for the different estimators of the two parameters and for all sample sizes are Tabulated and represented through some Figures. 
All simulation studies presented here are obtained via the MathCAD14 software. The MSE of the different estimators of $\alpha$ and $\lambda$ are reported in Tables (1-6) and described in Figures (1-18). From simulation study many observations can be made on the performance of PGPWMs estimators with doubly, left and right censored samples. These observations are summarized as follows:

1. It is observed from Tables (1),(2)and (3) that the MSE of estimators for $\alpha$ decreases as the sample size increases. This indicate that PGPWMs based on doubly, left and right censoring provided consistent estimators for $\alpha$ [see Figures(1-9)]

2. It is observed from Tables (4),(5)and (6) that the MSE of estimators for $\lambda$ decreases as the sample size increases. This indicates that PGPWMs with doubly, left and right censoring provided consistent estimators for $\lambda$ [see Figures (10-18)]

3. When the exponents of PGPWMs $u_{1}$ and $u_{2}$ decrease, the MSE of estimators for $\alpha$ and $\lambda$ decreases [see Figures (1-18) and Tables (1-5)]

4. Considering the MSE of the different estimators of $\alpha$, it is clear from Tables (1) (2)and (3) that the PGPWMs under right censoring estimator has the minimum MSE in almost all of the cases followed by PGPWMs under doubly censoring and the PGPWMs under left censoring [see Figures(1-9)]

5. Considering the MSE of the different estimators of $\lambda$, it is clear from Tables (4), (5)and (6) that the PGPWMs under right censoring estimator has the minimum MSE in almost all of the cases followed by PGPWMs under doubly censoring and PGPWMs under left censoring [see Figures (10-18)].

\section{Data Analysis}

This section presents a real data set for illustrative purposes. Lawless (1982) provided real data, which represents the numberof million revolutions before failure for each of 23 ball bearings in a life test: $17.88,28.92,33.00,41.52,42.12,45.60,48.80,51.84,51.96$, 54.12 , 55.56, 67.80, 68.64, 68.64, 68.88, 84.12, 93.12, 98.64, 105.12, 105.84, 127.92, 128.04 , and 173.40 .

Data analysis is presented to illustrate different PGWMs estimators from doubly, left and right censored samples. The data set is carried out to investigate the properties of the PGPWMs method of estimation for the EE distribution. The investigated properties of estimators for the two parameters $\alpha$ and $\lambda$ are biases and MSEs. The results are displayed in Tables 7 and 8 and some observations are summarized as follows:

1. Considering the MSE of the estimator for $\alpha$, it is clear from Table (7) that the PGPWMs under doubly censoring estimator has the minimum MSE in almost all of the cases considered for estimating $\alpha$ follow it PGPWMs under right censoring and the final PGPWMs under left censoring.

2. Considering the MSE of the estimator for $\lambda$, it is clear from Table (8) that the PGPWMs under doubly censoring estimator has the minimum MSE in almost all of the cases considered for estimating $\lambda$ follow it PGPWMs under right censoring and the final PGPWMs under left censoring. 


\section{Concluding Remarks}

In this study, the method of PGPWMs is introduced as a new method for estimating the parameters of distribution from censored sample. This framework covers the methods; PWMs, PPWMs and GPWMs. The GPWMs can be obtained via PGPWMs by setting the lower bound $\mathrm{c}=0$ and upper bound $\mathrm{d}=1$. Also, the PPWMs can be obtained from PGPWMs by setting the exponents of methods $u$ equal integer numbers. Finally, the PWMs can be obtained from PGPWMs by setting the exponents of methods $u$ equal integer numbers and the lower and upper bound equal 0 and 1 respectively

The method of PGPWMs has been applied for estimating the unknown parameters of EE distribution from doubly censored sample. This method has two special cases the left and right censored samples. All methods have provided consistent estimators for shape and scale parameters of EE distribution in terms of MSE. Comparing the performance of estimators from doubly, left and right censored samples the PGPWMs with right censored have the smallest MSE.

\section{References}

1. Al-Khodary, E., Hassan, A., and Allam, S. (2007). Self-determinant probability weighted moments method for estimating the parameters of the generalized exponential distribution. Proceeding of the $1^{9 s t}$ Annual Conference Statistics and Computer Modeling in Human and Social Sciences, Faculty of Economics and Political Science, Cairo University. 19, 159-176.

2. Al-Khodary, E., Hassan, A., and Allam, S. (2008). Double Censoring Partial Probability Weighted Moments Estimation of the Generalized Exponential Distribution, InterStat, pp. 1-24.

3. Deng, J. and Pandey, M.D. (2009). Using Partial Probability Weighted Moments and Partial Maximum entropy to estimate Quantiles from Censored Samples Probabilistic Engineering Mechanics, 24, 407- 417.

4. ElHaroun, N.M. (2009). Generalized Probability Weighted Moments Estimators for Some Distributions. M.Sc. Thesis, Cairo University.

5. Hosking, J. R. M. (1986). The Theory of Probability Weighted Moments. Research Report RC 12210, IBM Research Division, Yorktown Heights, NY.

6. Gupta, R.D. and Kundu, D. (1999). Generalized exponential distributions, Australian and New Zealand Journal of Statistics, 41(2), 173-188.

7. Lawless, J. F. (1982). Statistical Models and Methods for Lifetime Data, John Wiley \& Sons, New York

8. Rasmussen, P. (2001). Generalized Probability Weighted Moment: Application to the Generalized Pareto Distribution. Water Resources Research, 37, 6, 1745-1751.

9. Wang, Q.J. (1990a). Estimation of the GEV distribution from censored samples by method of partial probability weighted moments, Journal of Hydrology, 120 (1-4), 103-114. 
10. Wang, Q. J. (1990b). Unbiased estimation of probability weighted moments and partial probability weighted moments from systematic and historical flood information and their application to estimating the GEV distribution. Journal of Hydrology 120, 115-124.

11. Wang, Q. J. (1996). Using partial probability weighted moments to fit the extreme value distributions to censored samples. Water Resources Research, 32(6), 17671771.

12. Zafirakou-Koulouris, A., Vogel, R.M., Craig, S., M., Habermier, J. (1998). Lmoment Diagram for Censored Observation. Water Resources Research, 34, (5), 1241-1249. 


\section{Appendix}

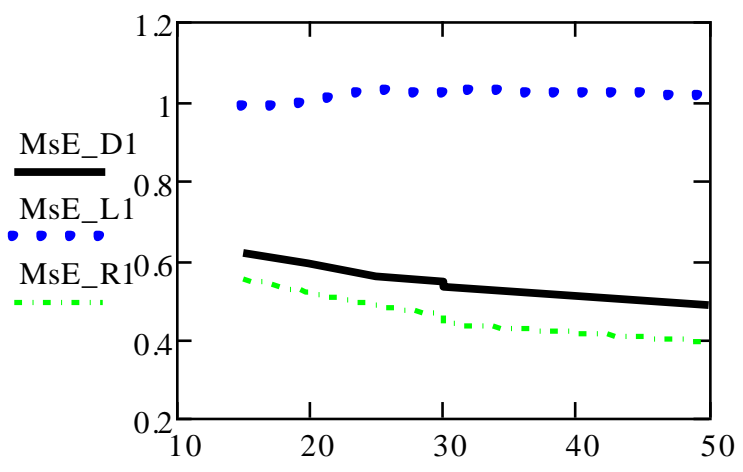

Figure1: The MSE of $\hat{\alpha}, \hat{\alpha}_{L}, \hat{\alpha}_{R}{ }^{\mathrm{n}}$ when $\mathrm{u}_{1}=0.1$ $\mathrm{u}_{2}=0.4$ for $\alpha=0.5$

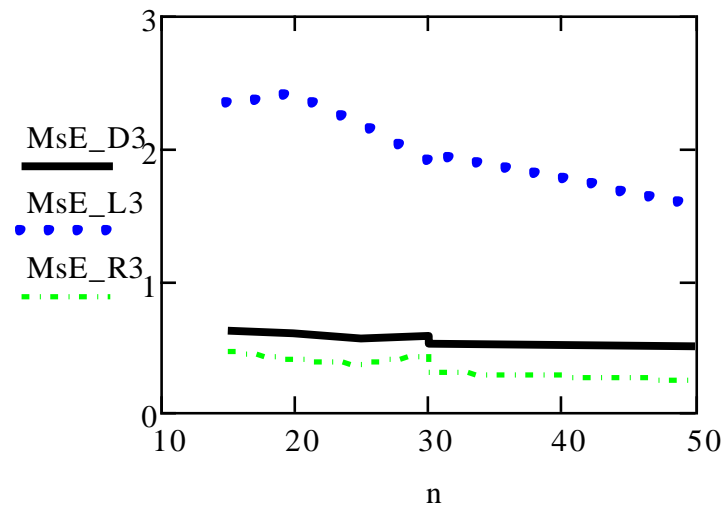

Figure3: The MSE of $\hat{\alpha}, \hat{\alpha}_{L}, \hat{\alpha}_{R}$ when $\mathrm{u}_{1}=0.1$, $\mathrm{u}_{2}=0.4$ for $\alpha=0.7$

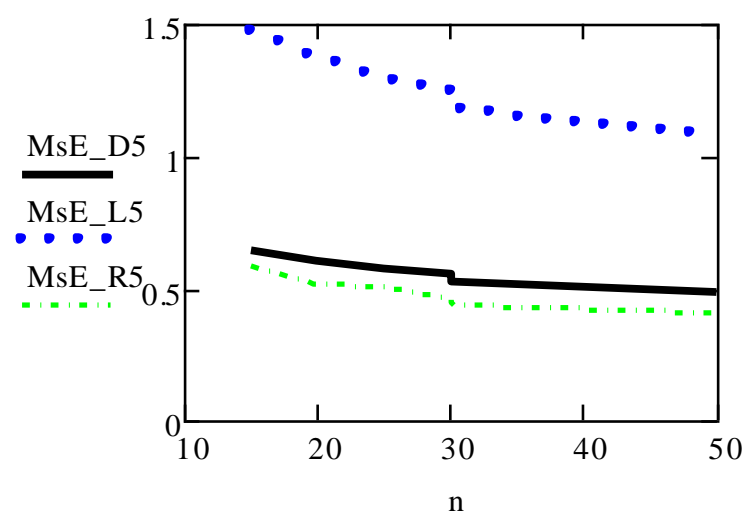

Figure5: The MSE of $\hat{\alpha}, \hat{\alpha}_{L}, \hat{\alpha}_{R}$ when $\mathrm{u}_{1}=0.5$ $\mathrm{u}_{2}=1.5$ for $\alpha=0.6$

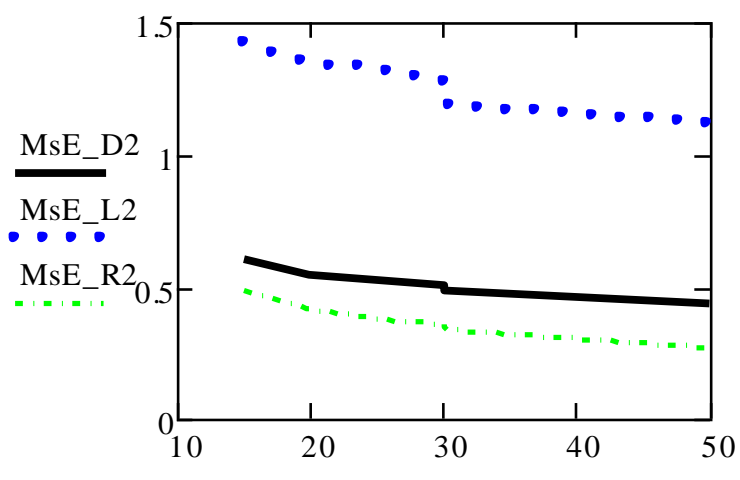

Figure2: The MSE of $\hat{\alpha}, \hat{\alpha}_{L}, \hat{\alpha}_{R}$ when $\mathrm{u}_{1}=0.1$, $\mathrm{u}_{2}=0.4$ for $\alpha=0.6$

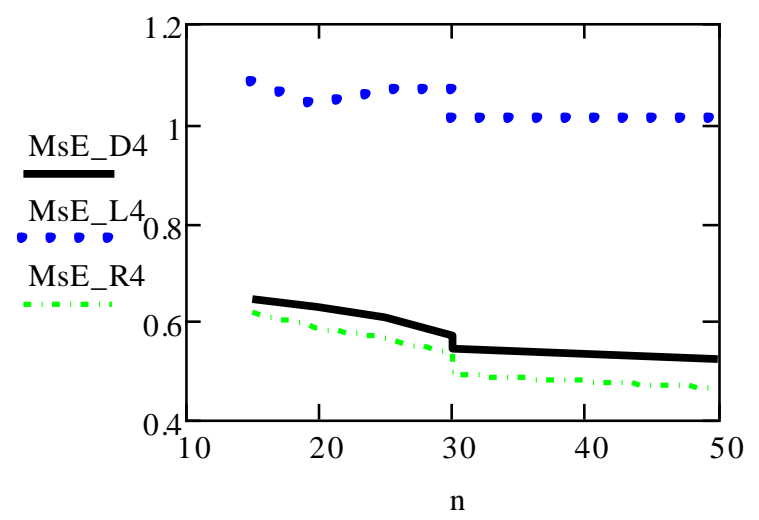

Figure4: The MSE of $\hat{\alpha}, \hat{\alpha}_{L}, \hat{\alpha}_{R}$ when $\mathrm{u}_{1}=0.5$ $\mathrm{u}_{2}=1.5$ for $\alpha=0.5$

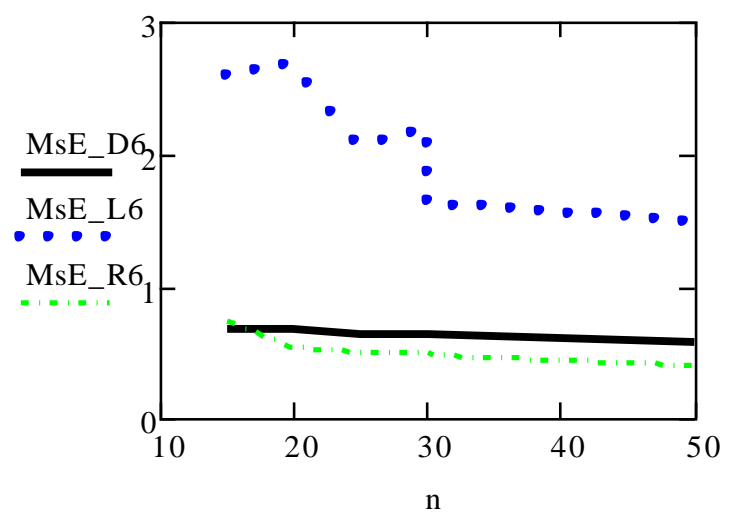

Figure6: The MSE of $\hat{\alpha}, \hat{\alpha}_{L}, \hat{\alpha}_{R}$ when $\mathrm{u}_{1}=0.5$ $\mathrm{u}_{2}=1.5$ for $\alpha=07$ 


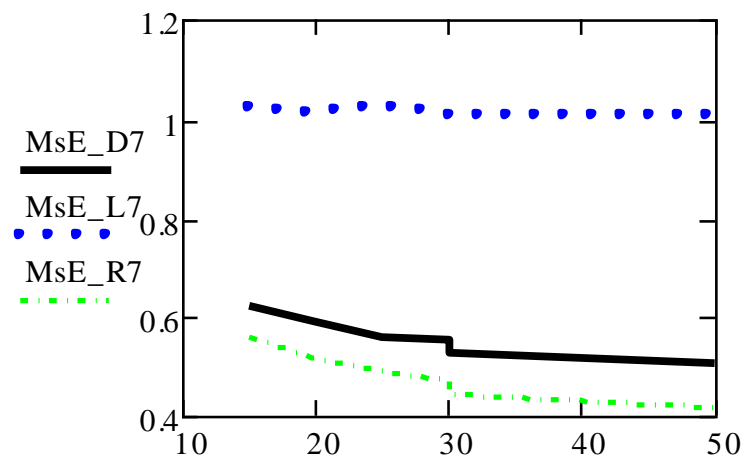

Figure7: The MSE of $\hat{\alpha}, \hat{\alpha}_{L}, \hat{\alpha}_{R}$ when $\mathrm{u}_{1}=0$ $\mathrm{u}_{2}=1$ for $\alpha=0.5$

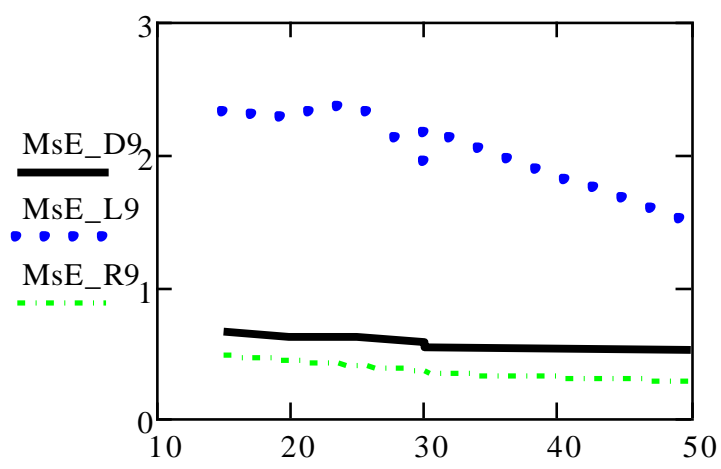

Figure9: The MSE of $\hat{\alpha}, \hat{\alpha}_{L}, \hat{\alpha}_{R}$ when $\mathrm{u}_{1}=0$ $\mathrm{u}_{2}=1$ for $\alpha=0.5$

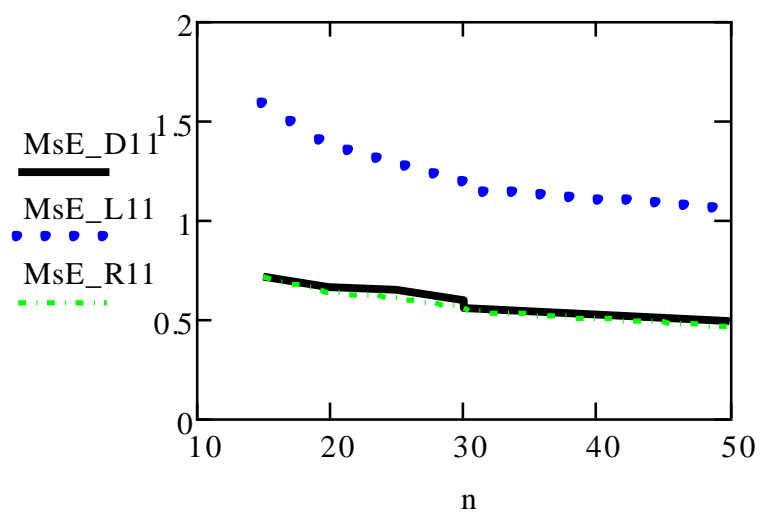

Figure 11: The MSE of $\hat{\lambda}, \hat{\lambda}_{L}, \hat{\lambda}_{R}$ when $\mathrm{u}_{1}=0.1$ $\mathrm{u}_{2}=1$ for $\alpha=0.6$

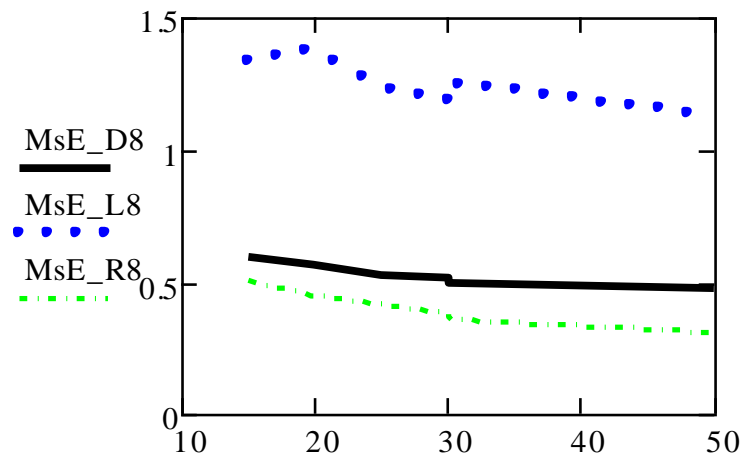

Figure8: The MSE of $\hat{\alpha}, \hat{\alpha}_{L}, \hat{\alpha}_{R}$ when $\mathrm{u}_{1}=0$ $\mathrm{u}_{2}=1$ for $\alpha=0.6$

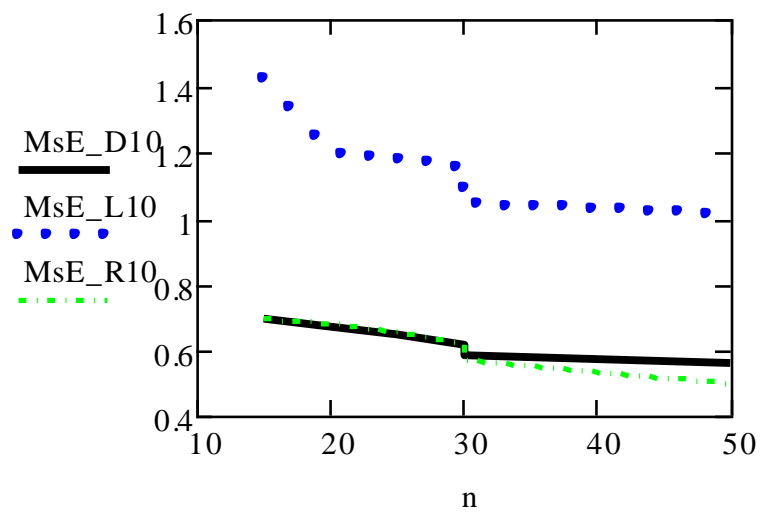

Figure10: The MSE of $\hat{\lambda}, \hat{\lambda}_{L}, \hat{\lambda}_{R}$ when $u_{1}=0.1$ $\mathrm{u}_{2}=1$ for $\alpha=0.5$

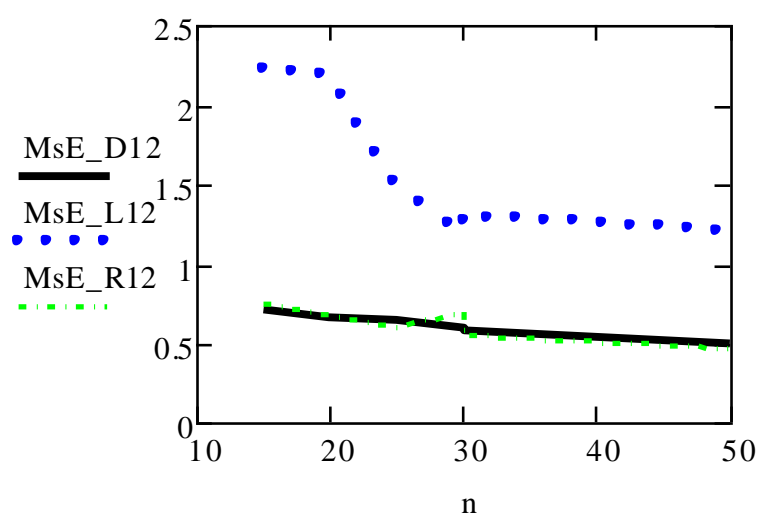

Figure12: The MSE of $\hat{\lambda}, \hat{\lambda}_{L}, \hat{\lambda}_{R}$ when $u_{1}=0.1$ $\mathrm{u}_{2}=1$ for $\alpha=0.7$ 


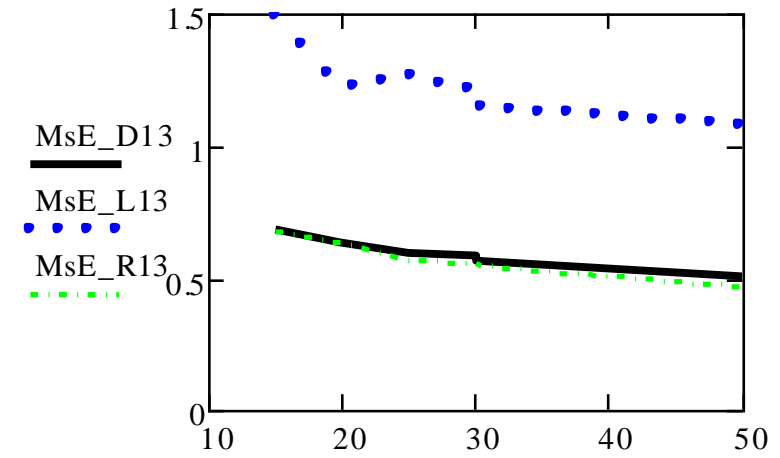

Figure13: The MSE of $\hat{\lambda}, \hat{\lambda}_{L}, \hat{\lambda}_{R}$ when $\mathrm{u}_{1}=0.1$ $\mathrm{u}_{2}=0.4$ for $\alpha=0.5$

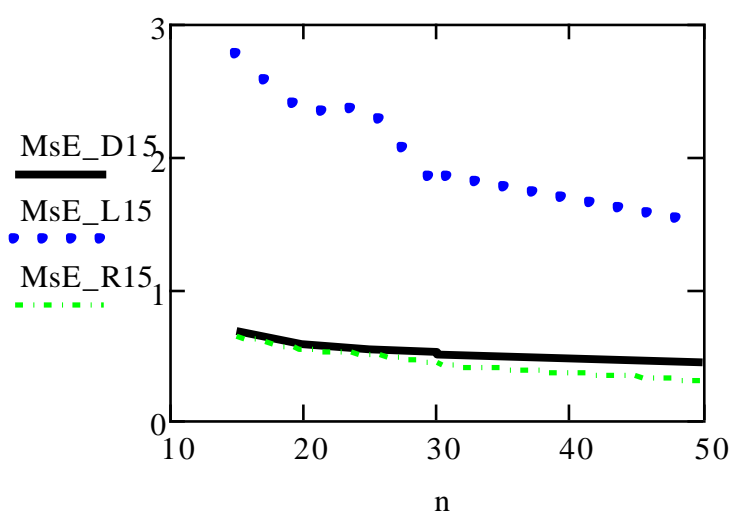

Figure 15: The MSE of $\hat{\lambda}, \hat{\lambda}_{L}, \hat{\lambda}_{R}$ when $\mathrm{u}_{1}=0.1$ $\mathrm{u}_{2}=0.4$ for $\alpha=0.7$

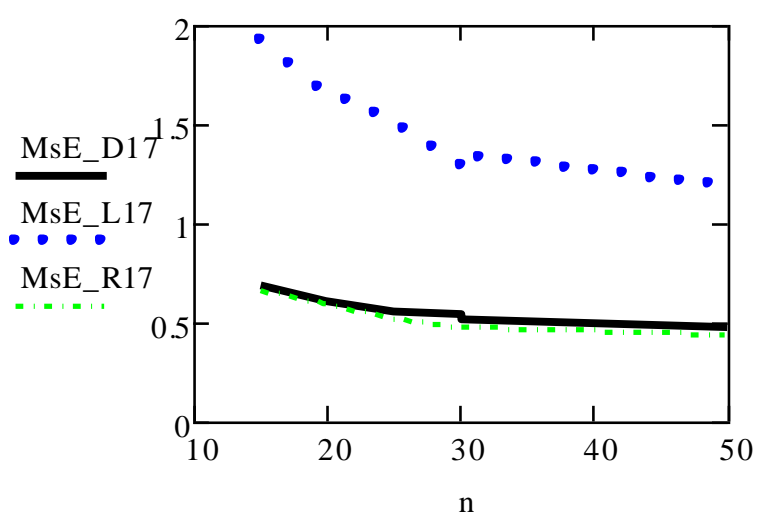

Figure 17: The MSE of $\hat{\lambda}, \hat{\lambda}_{L}, \hat{\lambda}_{R}$ when $\mathrm{u}_{1}=0.5$ $\mathrm{u}_{2}=1.5$ for $\alpha=0.6$

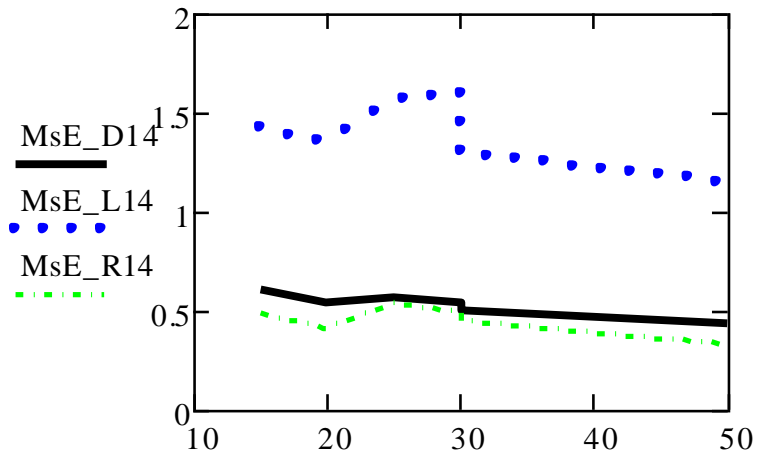

Figure 14: The MSE of $\hat{\lambda}, \hat{\lambda}_{L}, \hat{\lambda}_{R}$ when $\mathrm{u}_{1}=0.1$ $\mathrm{u}_{2}=0.4$ for $\alpha=0.6$

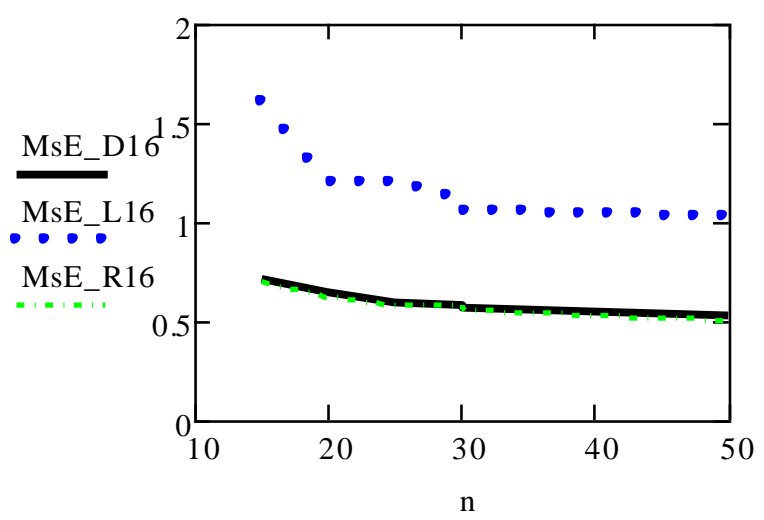

Figure16: The MSE of $\hat{\lambda}, \hat{\lambda}_{L}, \hat{\lambda}_{R}$ when $\mathrm{u}_{1}=0.5$ $\mathrm{u}_{2}=1.5$ for $\alpha=0.5$

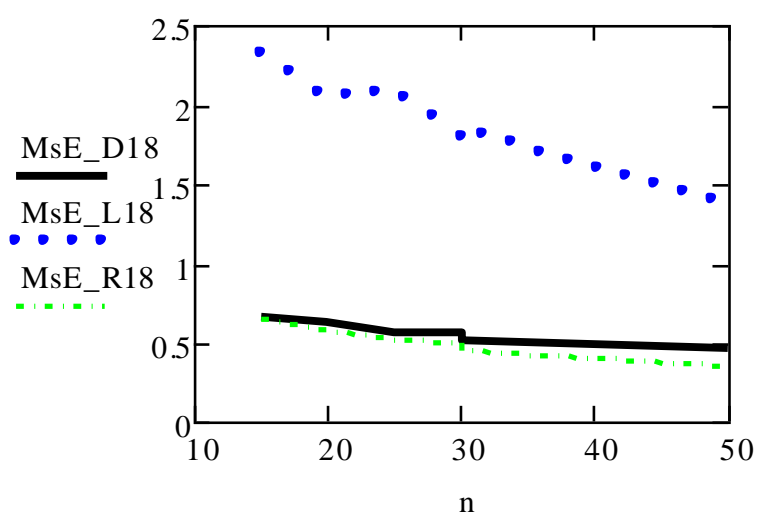

Figure 18: The MSE of $\hat{\lambda}, \hat{\lambda}_{L}, \hat{\lambda}_{R}$ when $\mathrm{u}_{1}=0.5$ $\mathrm{u}_{2}=1.5$ for $\alpha=0.7$ 
Table 1: Mean, Bias and MSE of PGPWMs Parameter Estimators for EE distribution from Doubly, Left and Right Censoring for $u_{1}=0.5$ and $u_{2}=1.5$

\begin{tabular}{|c|c|c|c|c|c|c|c|c|c|c|}
\hline \multirow{2}{*}{$\begin{array}{l}\text { Sample } \\
\text { size } n\end{array}$} & \multirow{2}{*}{$\begin{array}{l}\text { Properties } \\
\text { of } \alpha\end{array}$} & \multicolumn{3}{|c|}{ PGPWMs of $\hat{\alpha}$} & \multicolumn{3}{|c|}{ LPPWMs of $\hat{\alpha}_{L}$} & \multicolumn{3}{|c|}{ RPPWMs of $\hat{\alpha}_{R}$} \\
\hline & & $\begin{array}{c}\alpha \\
=0.5\end{array}$ & $\begin{array}{c}\alpha \\
=0.6\end{array}$ & $\begin{array}{c}\alpha \\
=0.7\end{array}$ & $\begin{array}{c}\alpha \\
=0.5\end{array}$ & $\begin{array}{c}\alpha \\
=0.6\end{array}$ & $\begin{array}{c}\alpha \\
=0.7\end{array}$ & $\begin{array}{c}\alpha \\
=0.5\end{array}$ & $\begin{array}{c}\alpha \\
=0.6\end{array}$ & $\begin{array}{c}\alpha \\
=0.7\end{array}$ \\
\hline \multirow{3}{*}{15} & Mean & 0.277 & 0.349 & 0.425 & 0.137 & 0.281 & 0.464 & 0.290 & 0.369 & 0.436 \\
\hline & Bias & -0.723 & -0.651 & -0.575 & -0.863 & -0.719 & -0.536 & -0.710 & -0.631 & -0.564 \\
\hline & MSE & 0.644 & 0.637 & 0.664 & 1.078 & 1.466 & 2.581 & 0.614 & 0.582 & 0.725 \\
\hline \multirow{3}{*}{20} & Mean & 0.296 & 0.417 & 0.489 & 0.078 & 0.196 & 0.458 & 0.318 & 0.440 & 0.519 \\
\hline & Bias & -0.704 & -0.583 & -0.511 & -0.922 & -0.804 & -0.542 & -0.682 & -0.560 & -0.481 \\
\hline & MSE & 0.625 & 0.598 & 0.662 & 1.034 & 1.369 & 2.683 & 0.586 & 0.517 & 0.537 \\
\hline \multirow{3}{*}{25} & Mean & 0.316 & 0.437 & 0.555 & 0.077 & 0.136 & 0.278 & 0.340 & 0.439 & 0.571 \\
\hline & Bias & -0.684 & -0.563 & -0.445 & -0.923 & -0.864 & -0.722 & -0.660 & -0.561 & -0.429 \\
\hline & MSE & 0.606 & 0.574 & 0.627 & 1.061 & 1.292 & 2.053 & 0.561 & 0.508 & 0.494 \\
\hline \multirow{3}{*}{30} & Mean & 0.348 & 0.489 & 0.601 & 0.058 & 0.102 & 0.265 & 0.361 & 0.505 & 0.597 \\
\hline & Bias & -0.652 & -0.511 & -0.399 & -0.942 & -0.898 & -0.735 & -0.639 & -0.495 & -0.403 \\
\hline & MSE & 0.570 & 0.555 & 0.626 & 1.063 & 1.240 & 2.172 & 0.532 & 0.456 & 0.484 \\
\hline \multirow{3}{*}{35} & Mean & 0.388 & 0.490 & 0.601 & 0.018 & 0.069 & 0.144 & 0.407 & 0.525 & 0.617 \\
\hline & Bias & -0.612 & -0.510 & -0.399 & -0.982 & -0.931 & -0.856 & -0.593 & -0.475 & -0.383 \\
\hline & MSE & 0.540 & 0.521 & 0.627 & 1.004 & 1.172 & 1.625 & 0.489 & 0.433 & 0.464 \\
\hline \multirow{3}{*}{50} & Mean & 0.405 & 0.533 & 0.688 & 0.003 & 0.024 & 0.098 & 0.442 & 0.544 & 0.724 \\
\hline & Bias & -0.595 & -0.467 & -0.312 & -0.997 & -0.976 & -0.902 & -0.558 & -0.456 & -0.276 \\
\hline & MSE & 0.519 & 0.482 & 0.579 & 1.003 & 1.074 & 1.482 & 0.462 & 0.400 & 0.389 \\
\hline
\end{tabular}

Table 2: Mean, Bias and MSE of PGPWMs Parameter Estimators for EE distribution from Doubly, Left and Right Censoring for $u_{1}=0.1$ and $u_{2}=0.4$

\begin{tabular}{|c|c|c|c|c|c|c|c|c|c|c|}
\hline \multirow[t]{2}{*}{$\begin{array}{l}\text { Sample } \\
\text { size } n\end{array}$} & \multirow[t]{2}{*}{$\begin{array}{l}\text { Properties } \\
\text { of } \alpha\end{array}$} & \multicolumn{3}{|c|}{ PGPWMs of $\hat{\alpha}$} & \multicolumn{3}{|c|}{ LPPWMs of $\hat{\alpha}_{L}$} & \multicolumn{3}{|c|}{ RPPWMs of $\hat{\alpha}_{R}$} \\
\hline & & $\alpha=0.5$ & $\alpha=0.6$ & $\alpha=0.7$ & $\alpha=0.5$ & $\alpha=0.6$ & $\alpha=0.7$ & $\alpha=0.5$ & $\alpha=0.6$ & $\alpha=0.7$ \\
\hline \multirow{3}{*}{15} & Mean & 0.300 & 0.366 & 0.473 & 0.196 & 0.400 & 0.639 & 0.330 & 0.431 & 0.503 \\
\hline & Bias & 0.700 & -0.634 & -0.527 & -0.804 & -0.600 & -0.361 & -0.670 & -0.569 & -0.497 \\
\hline & MSE & 0.614 & 0.600 & 0.617 & 0.981 & 1.422 & 2.320 & 0.550 & 0.481 & 0.455 \\
\hline \multirow{3}{*}{20} & Mean & 0.320 & 0.421 & 0.543 & 0.108 & 0.294 & 0.557 & 0.367 & 0.497 & 0.568 \\
\hline & Bias & -0.680 & -0.579 & -0.457 & -0.892 & -0.706 & -0.443 & -0.633 & -0.503 & -0.432 \\
\hline & MSE & 0.588 & 0.543 & 0.588 & 0.992 & 1.340 & 2.389 & 0.519 & 0.410 & 0.394 \\
\hline \multirow{3}{*}{25} & Mean & 0.361 & 0.467 & 0.584 & 0.096 & 0.269 & 0.455 & 0.390 & 0.525 & 0.629 \\
\hline & Bias & -0.639 & -0.533 & -0.416 & -0.904 & -0.731 & -0.545 & -0.610 & -0.475 & -0.371 \\
\hline & MSE & 0.556 & 0.520 & 0.549 & 1.02 & 1.321 & 2.178 & 0.482 & 0.379 & 0.347 \\
\hline \multirow{3}{*}{30} & Mean & 0.376 & 0.500 & 0.640 & 0.070 & 0.230 & 0.346 & 0.413 & 0.553 & 0.660 \\
\hline & Bias & -0.624 & -0.500 & -0.360 & $\begin{array}{l}-0.93 \\
\end{array}$ & -0.770 & -0.654 & -0.587 & -0.447 & -0.340 \\
\hline & MSE & 0.540 & 0.503 & 0.564 & 1.018 & 1.276 & 1.890 & 0.455 & 0.351 & 0.430 \\
\hline \multirow{3}{*}{35} & Mean & 0.383 & 0.506 & 0.631 & 0.067 & 0.128 & 0.368 & 0.429 & 0.573 & 0.661 \\
\hline & Bias & -0.617 & -0.494 & -0.369 & -0.933 & -0.872 & -0.632 & -0.571 & -0.427 & -0.339 \\
\hline & MSE & 0.528 & 0.484 & 0.510 & 1.022 & 1.186 & 1.956 & 0.434 & 0.331 & 0.302 \\
\hline \multirow{3}{*}{50} & Mean & 0.433 & 0.627 & 0.730 & 0.020 & 0.070 & 0.192 & 0.467 & 0.631 & 0.728 \\
\hline & Bias & -0.567 & -0.373 & -0.270 & -0.980 & -0.930 & -0.808 & -0.533 & -0.369 & -0.272 \\
\hline & MSE & 0.486 & 0.432 & 0.484 & 1.012 & 1.111 & 1.560 & 0.391 & 0.262 & 0.236 \\
\hline
\end{tabular}


Table 3: Mean, Bias and MSE of the PPWMs Parameter Estimators for EE distribution from Doubly, Left and Right Censoring when $u_{1}=0$ and

$$
u_{2}=1
$$

\begin{tabular}{|c|c|c|c|c|c|c|c|c|c|c|}
\hline \multirow{2}{*}{$\begin{array}{l}\text { Sample } \\
\text { size } n\end{array}$} & \multirow{2}{*}{$\begin{array}{l}\text { Properties } \\
\text { of } \alpha\end{array}$} & \multicolumn{3}{|c|}{ PGPWMs of $\hat{\alpha}$} & \multicolumn{3}{|c|}{ LPPWMs of $\hat{\alpha}_{L}$} & \multicolumn{3}{|c|}{ RPPWMs of $\hat{\alpha}_{R}$} \\
\hline & & $\alpha=0.5$ & $\alpha=0.6$ & $\alpha=0.7$ & $\alpha=0.5$ & $\alpha=0.6$ & $\alpha=0.7$ & $\alpha=0.5$ & $\alpha=0.6$ & $\alpha=0.7$ \\
\hline \multirow{3}{*}{15} & Mean & 0.298 & 0.387 & 0.508 & 0.197 & 0.352 & 0.524 & 0.330 & 0.409 & 0.519 \\
\hline & Bias & -0.702 & -0.613 & -0.492 & -0.803 & -0.648 & -0.476 & -0.670 & -0.591 & -0.481 \\
\hline & MSE & 0.619 & 0.593 & 0.650 & 1.022 & 1.337 & 2.316 & 0.557 & 0.506 & 0.473 \\
\hline \multirow{3}{*}{20} & Mean & 0.326 & 0.423 & 0.543 & 0.103 & 0.268 & 0.430 & 0.367 & 0.468 & 0.552 \\
\hline & Bias & -0.674 & -0.577 & -0.457 & -0.897 & -0.732 & -0.570 & -0.633 & -0.532 & -0.448 \\
\hline & MSE & 0.589 & 0.561 & 0.618 & 1.011 & 1.372 & 2.276 & 0.515 & 0.448 & 0.432 \\
\hline \multirow{3}{*}{25} & Mean & 0.358 & 0.485 & 0.599 & 0.079 & 0.185 & 0.455 & 0.388 & 0.503 & 0.604 \\
\hline & Bias & -0.642 & -0.515 & -0.401 & -0.921 & -0.815 & -0.545 & -0.612 & -0.497 & -0.396 \\
\hline & MSE & 0.559 & 0.521 & 0.609 & 1.024 & 1.233 & 2.374 & 0.488 & 0.413 & 0.388 \\
\hline \multirow{3}{*}{30} & Mean & 0.366 & 0.506 & 0.610 & 0.043 & 0.130 & 0.322 & 0.409 & 0.546 & 0.644 \\
\hline & Bias & -0.634 & -0.494 & -0.390 & -0.957 & -0.817 & -0.678 & -0.591 & -0.454 & -0.356 \\
\hline & MSE & 0.553 & 0.515 & 0.572 & 1.007 & 1.183 & 1.941 & 0.470 & 0.378 & 0.358 \\
\hline \multirow{3}{*}{35} & Mean & 0.389 & 0.525 & 0.653 & 0.026 & 0.148 & 0.346 & 0.431 & 0.565 & 0.675 \\
\hline & Bias & -0.611 & -0.475 & -0.347 & -0.974 & -0.852 & -0.654 & -0.569 & -0.435 & -0.325 \\
\hline & MSE & 0.525 & 0.491 & 0.542 & 1.004 & 1.251 & 2.180 & 0.441 & 0.351 & 0.331 \\
\hline \multirow{3}{*}{50} & Mean & 0.417 & 0.547 & 0.736 & 0.008 & 0.067 & 0.142 & 0.456 & 0.627 & 0.72 \\
\hline & Bias & -0.583 & -0.453 & -0.264 & -0.992 & -0.933 & -0.858 & -0.544 & -0.373 & -0.280 \\
\hline & MSE & 0.503 & 0.469 & 0.515 & 1.007 & 1.123 & 1.480 & 0.416 & 0.301 & 0.273 \\
\hline
\end{tabular}

Table 4: Mean, Bias and MSE of PGPWMs Parameter Estimators for EE distribution from Doubly, Left and Right Censoring for $u_{1}=0.5$ and $u_{2}=1.5$

\begin{tabular}{|c|c|c|c|c|c|c|c|c|c|c|}
\hline \multirow{2}{*}{$\begin{array}{l}\text { Sample } \\
\text { size } n\end{array}$} & \multirow{2}{*}{$\begin{array}{c}\text { Properties } \\
\text { of } \lambda\end{array}$} & \multicolumn{3}{|c|}{ PGPWMs of $\hat{\lambda}$} & \multicolumn{3}{|c|}{ LPPWMs of $\hat{\lambda}_{L}$} & \multicolumn{3}{|c|}{ RPPWMs of $\hat{\lambda}_{R}$} \\
\hline & & $\alpha=0.5$ & $\alpha=0.6$ & $\alpha=0.7$ & $\alpha=0.5$ & $\alpha=0.6$ & $\alpha=0.7$ & $\alpha=0.5$ & $\alpha=0.6$ & $\alpha=0.7$ \\
\hline \multirow{3}{*}{15} & Mean & 0.259 & 0.331 & 0.394 & 0.211 & 0.334 & 0.451 & 0.279 & 0.367 & 0.446 \\
\hline & Bias & -0.741 & -0.669 & -0.606 & -0.789 & -0.666 & -0.549 & -0.741 & -0.669 & -0.606 \\
\hline & MSE & 0.696 & 0.712 & 0.704 & 1.419 & 1.585 & 2.227 & 0.695 & 0.706 & 0.733 \\
\hline \multirow{3}{*}{20} & Mean & 0.281 & 0.395 & 0.436 & 0.124 & 0.214 & 0.428 & 0.311 & 0.435 & 0.501 \\
\hline & Bias & -0.719 & -0.605 & -0.564 & -0.874 & -0.786 & -0.572 & -0.719 & -0.605 & -0.564 \\
\hline & MSE & 0.672 & 0.660 & 0.66 & 1.194 & 1.366 & 2.181 & 0.675 & 0.634 & 0.676 \\
\hline \multirow{3}{*}{25} & Mean & 0.295 & 0.418 & 0.504 & 0.101 & 0.142 & 0.237 & 0.328 & 0.439 & 0.555 \\
\hline & Bias & -0.705 & -0.582 & -0.496 & -0.899 & -0.858 & -0.763 & -0.705 & -0.582 & -0.496 \\
\hline & MSE & 0.648 & 0.641 & 0.640 & 1.170 & 1.280 & 1.487 & 0.651 & 0.599 & 0.599 \\
\hline \multirow{3}{*}{30} & Mean & 0.322 & 0.452 & 0.545 & 0.075 & 0.097 & 0.215 & 0.345 & 0.494 & 0.578 \\
\hline & Bias & -0.678 & -0.548 & -0.455 & -0.925 & -0.903 & -0.785 & -0.678 & -0.548 & -0.455 \\
\hline & MSE & 0.615 & 0.589 & 0.588 & 1.153 & 1.186 & 1.596 & 0.611 & 0.558 & 0.693 \\
\hline \multirow{3}{*}{35} & Mean & 0.372 & 0.465 & 0.544 & 0.029 & 0.068 & 0.115 & 0.404 & 0.524 & 0.600 \\
\hline & Bias & -0.628 & -0.535 & -0.456 & -0.971 & -0.932 & -0.885 & -0.628 & -0.535 & -0.456 \\
\hline & MSE & 0.578 & 0.549 & 0.583 & 1.039 & 1.147 & 1.304 & 0.566 & 0.538 & 0.545 \\
\hline \multirow{3}{*}{50} & Mean & 0.388 & 0.500 & 0.619 & 0.003 & 0.021 & 0.071 & 0.33 & 0.535 & 0.706 \\
\hline & Bias & -0.612 & -0.500 & -0.381 & -0.997 & -0.979 & -0.929 & -0.612 & -0.500 & -0.381 \\
\hline & MSE & 0.555 & 0.492 & 0.499 & 1.005 & 1.048 & 1.206 & 0.493 & 0.465 & 0.468 \\
\hline
\end{tabular}


Table 5: Mean, Bias and MSE of PGPWMs Parameter Estimators for EE distribution from Doubly, Left and Right Censoring for $u_{1}=0.1$ and $u_{2}=0.4$

\begin{tabular}{|c|c|c|c|c|c|c|c|c|c|c|}
\hline \multirow[t]{2}{*}{$\begin{array}{l}\text { Sample } \\
\text { size } n\end{array}$} & \multirow{2}{*}{$\begin{array}{c}\text { Properties } \\
\text { of } \lambda\end{array}$} & \multicolumn{3}{|c|}{ PGPWMs of $\hat{\lambda}$} & \multicolumn{3}{|c|}{ LPPWMs of $\hat{\lambda}_{L}$} & \multicolumn{3}{|c|}{ RPPWMs of $\hat{\lambda}_{R}$} \\
\hline & & $\alpha=0.5$ & $\alpha=0.6$ & $\alpha=0.7$ & $\alpha=0.5$ & $\alpha=0.6$ & $\alpha=0.7$ & $\alpha=0.5$ & $\alpha=0.6$ & $\alpha=0.7$ \\
\hline \multirow{3}{*}{15} & Mean & 0.290 & 0.352 & 0.443 & 0.352 & 0.400 & 0.782 & 0.329 & 0.431 & 0.514 \\
\hline & Bias & -0.710 & -0.634 & -0.557 & -0.648 & -0.600 & -0.218 & -0.710 & -0.569 & -0.557 \\
\hline & MSE & 0.685 & 0.600 & 0.672 & 1.493 & 1.422 & 2.773 & 0.669 & 0.481 & 0.637 \\
\hline \multirow{3}{*}{20} & Mean & 0.307 & 0.421 & 0.496 & 0.179 & 0.294 & 0.594 & 0.361 & 0.497 & 0.572 \\
\hline & Bias & -0.693 & -0.579 & -0.504 & -0.821 & -0.706 & -0.406 & -0.697 & -0.503 & -0.504 \\
\hline & MSE & 0.634 & 0.543 & 0.582 & 1.210 & 1.340 & 2.329 & 0.631 & 0.410 & 0.535 \\
\hline \multirow{3}{*}{25} & Mean & 0.353 & 0.447 & 0.538 & 0.157 & 0.332 & 0.504 & 0.391 & 0.539 & 0.638 \\
\hline & Bias & -0.647 & -0.553 & -0.462 & -0.843 & -0.668 & -0.496 & -0.647 & -0.553 & -0.462 \\
\hline & MSE & 0.592 & 0.565 & 0.542 & 1.261 & 1.563 & 2.339 & 0.563 & 0.536 & 0.488 \\
\hline \multirow{3}{*}{30} & Mean & 0.366 & 0.489 & 0.588 & 0.114 & 0.300 & 0.353 & 0.414 & 0.576 & 0.669 \\
\hline & Bias & -0.634 & -0.511 & -0.412 & -0.886 & -0.700 & -0.647 & -0.634 & -0.511 & -0.412 \\
\hline & MSE & 0.578 & 0.542 & 0.517 & 1.200 & 1.597 & 1.770 & 0.549 & 0.483 & 0.430 \\
\hline \multirow{3}{*}{35} & Mean & 0.371 & 0.494 & 0.585 & 0.099 & 0.151 & 0.376 & 0.432 & 0.586 & 0.669 \\
\hline & Bias & -0.629 & -0.506 & -0.415 & -0.901 & -0.849 & -0.624 & -0.629 & -0.506 & -0.415 \\
\hline & MSE & 0.560 & 0.505 & 0.493 & 1.145 & 1.286 & 1.862 & 0.538 & 0.449 & 0.413 \\
\hline \multirow{3}{*}{50} & Mean & 0.420 & 0.595 & 0.681 & 0.032 & 0.078 & 0.192 & 0.466 & 0.644 & 0.743 \\
\hline & Bias & -0.580 & -0.405 & -0.319 & -0.968 & -0.922 & -0.808 & -0.580 & -0.405 & -0.319 \\
\hline & MSE & 0.508 & 0.43 & 0.436 & 1.072 & 1.142 & 1.485 & 0.465 & 0.328 & 0.305 \\
\hline
\end{tabular}

Table 6: Mean, Bias and MSE of PPWMs Parameter Estimators for EE distribution from Doubly, Left and Right Censoring for $u_{1}=0$ and $u_{2}=1$

\begin{tabular}{|c|c|c|c|c|c|c|c|c|c|c|}
\hline \multirow{2}{*}{$\begin{array}{l}\text { Sample } \\
\text { size } n\end{array}$} & \multirow{2}{*}{$\begin{array}{c}\text { Properties } \\
\text { of } \lambda\end{array}$} & \multicolumn{3}{|c|}{ PGPWMs of $\hat{\lambda}$} & \multicolumn{3}{|c|}{ LPPWMs of $\hat{\lambda}_{L}$} & \multicolumn{3}{|c|}{ RPPWMs of $\hat{\lambda}_{R}$} \\
\hline & & $\alpha=0.5$ & $\alpha=0.6$ & $\alpha=0.7$ & $\alpha=0.5$ & $\alpha=0.6$ & $\alpha=0.7$ & $\alpha=0.5$ & $\alpha=0.6$ & $\alpha=0.7$ \\
\hline \multirow{3}{*}{15} & Mean & 0.286 & 0.367 & 0.472 & 0.343 & 0.497 & 0.562 & 0.328 & 0.415 & 0.535 \\
\hline & Bias & -0.714 & -0.633 & -0.528 & -0.657 & -0.503 & -0.437 & -0.714 & -0.633 & -0.528 \\
\hline & MSE & 0.707 & 0.686 & 0.662 & 1.601 & 1.923 & 2.219 & 0.694 & 0.659 & 0.644 \\
\hline \multirow{3}{*}{20} & Mean & 0.311 & 0.400 & 0.502 & 0.162 & 0.332 & 0.442 & 0.359 & 0.469 & 0.561 \\
\hline & Bias & -0.689 & -0.600 & -0.498 & -0.838 & -0.668 & -0.558 & -0.689 & -0.600 & -0.498 \\
\hline & MSE & 0.641 & 0.606 & 0.633 & 1.193 & 1.653 & 2.043 & 0.625 & 0.593 & 0.571 \\
\hline \multirow{3}{*}{25} & Mean & 0.337 & 0.454 & 0.531 & 0.124 & 0.243 & 0.442 & 0.381 & 0.505 & 0.600 \\
\hline & Bias & -0.663 & -0.546 & -0.469 & -0.876 & -0.757 & -0.558 & -0.663 & -0.546 & -0.469 \\
\hline & MSE & 0.596 & 0.553 & 0.564 & 1.201 & 1.514 & 2.085 & 0.583 & 0.519 & 0.517 \\
\hline \multirow{3}{*}{30} & Mean & 0.350 & 0.481 & 0.566 & 0.070 & 0.158 & 0.328 & 0.409 & 0.547 & 0.656 \\
\hline & Bias & -0.650 & -0.519 & -0.434 & -0.093 & -0.842 & -0.672 & -0.650 & -0.519 & -0.434 \\
\hline & MSE & 0.585 & 0.542 & 0.556 & 1.104 & 1.291 & 1.796 & 0.578 & 0.480 & 0.489 \\
\hline \multirow{3}{*}{35} & Mean & 0.371 & 0.494 & 0.600 & 0.040 & 0.168 & 0.326 & 0.428 & 0.566 & 0.685 \\
\hline & Bias & -0.629 & -0.506 & -0.400 & -0.960 & -0.832 & -0.674 & -0.629 & -0.506 & -0.400 \\
\hline & MSE & 0.562 & 0.515 & 0.514 & 1.050 & 1.345 & 1.834 & 0.547 & 0.468 & 0.452 \\
\hline \multirow{3}{*}{50} & Mean & 0.405 & 0.519 & 0.682 & 0.012 & 0.077 & 0.135 & 0.457 & 0.637 & 0.733 \\
\hline & Bias & -0.595 & -0.481 & -0.318 & -0.988 & -0.923 & -0.865 & -0.595 & -0.481 & -0.318 \\
\hline & MSE & 0.527 & 0.476 & 0.463 & 1.025 & 1.183 & 1.367 & 0.499 & 0.434 & 0.341 \\
\hline
\end{tabular}


Table 7: Mean, Bias and MSE of the shape parameter estimators for EE distribution by the methods of PGPWMs with doubly, left and right censored based on real data

\begin{tabular}{|c|c|c|c|c|c|c|}
\hline \multirow{2}{*}{$\begin{array}{l}\text { Sample } \\
\text { size } n\end{array}$} & \multirow{2}{*}{$\begin{array}{l}\text { Properties } \\
\text { Of } \alpha\end{array}$} & \multirow[b]{2}{*}{$u_{1}$} & \multirow[b]{2}{*}{$u_{2}$} & \multicolumn{3}{|c|}{$\alpha=5.28$} \\
\hline & & & & PGPWMs of $\hat{\alpha}$ & LPGPWMs of $\hat{\alpha}$ & RPGPWMs of $\hat{\alpha}$ \\
\hline \multirow{9}{*}{23} & Mean & \multirow{3}{*}{0.5} & \multirow{3}{*}{1.5} & 0.8948 & 1.1401 & 0.9034 \\
\hline & Bias & & & 0.3943 & 0.6401 & 0.4034 \\
\hline & MSE & & & 0.1554 & 0.4098 & 0.1627 \\
\hline & Mean & \multirow{3}{*}{0.1} & \multirow{3}{*}{0.4} & 0.8308 & 1.0817 & 0.9472 \\
\hline & Bias & & & 0.3308 & 0.5817 & 0.4472 \\
\hline & MSE & & & 0.1094 & 0.3384 & 0.2000 \\
\hline & Mean & \multirow{3}{*}{0} & \multirow{3}{*}{1} & 0.9383 & 1.2248 & 1.0088 \\
\hline & Bias & & & 0.4383 & 0.7248 & 0.5088 \\
\hline & MSE & & & 0.1921 & 0.5254 & 0.2589 \\
\hline
\end{tabular}

Table 8: Mean, Bias and MSE of the scale parameter estimators for EE distribution by the methods of PGPWMs with doubly, left and right censored based on real data

\begin{tabular}{|c|c|c|c|c|c|c|}
\hline \multirow{2}{*}{$\begin{array}{l}\text { Sample } \\
\text { size } n\end{array}$} & \multirow{2}{*}{$\begin{array}{l}\text { Properties } \\
\text { Of } \alpha\end{array}$} & \multirow[b]{2}{*}{$u_{1}$} & \multirow[b]{2}{*}{$u_{2}$} & \multicolumn{3}{|c|}{$\lambda=1$} \\
\hline & & & & PGPWMs of $\hat{\lambda}$ & LPGPWMs of $\hat{\lambda}$ & RPGPWMs of $\hat{\lambda}$ \\
\hline \multirow{9}{*}{23} & Mean & \multirow{3}{*}{0.5} & \multirow{3}{*}{1.5} & 0.0320 & 0.0263 & 0.0314 \\
\hline & Bias & & & -0.9680 & -0.9688 & -0.9686 \\
\hline & MSE & & & 0.9370 & 0.9386 & 0.9382 \\
\hline & Mean & \multirow{3}{*}{0.1} & \multirow{3}{*}{0.4} & 0.1547 & 0.0279 & 0.0282 \\
\hline & Bias & & & -0.8453 & -0.9664 & -0.9659 \\
\hline & MSE & & & 0.7145 & 0.9339 & 0.9300 \\
\hline & Mean & \multirow{3}{*}{0} & \multirow{3}{*}{1} & 0.0261 & 0.0098 & 0.0211 \\
\hline & Bias & & & -0.9690 & -0.9902 & -0.9761 \\
\hline & MSE & & & 0.9390 & 0.9805 & 0.9527 \\
\hline
\end{tabular}

